\title{
Non Blind Image Watermarking Scheme using DWT, FWHT and SVD for Image Authentication
}

\author{
Dawinder Singh \\ Research Scholar, CE Section \\ Yadawindera College of Engineering \\ Punjabi University, Guru Kashi Campus Talwandi \\ Sabo, 151302, Punjab, India
}

\author{
Manoj Kumar \\ Assistant professor, CE Section \\ Yadawindera College of Engineering \\ Punjabi University, Guru Kashi Campus Talwandi \\ Sabo, 151302, Punjab, India
}

\begin{abstract}
In this paper, a watermarking scheme has been proposed for color images using SVD,DWT and FWHT which enhanced the embedding capacity and imperceptibility of watermarked image. We used 1024*1024 cover images and 256*256 watermark image. We have embedded four watermarks into single cover image and to measure efficiency of proposed scheme, parameters have been used such as Peak Signal to Noise Ratio (PSNR) and the Normalized Correlation (NC). Watermarked images have been exposed to several noise adding attacks to check robustness of given algorithm.
\end{abstract}

\section{Keywords}

DWT; SVD; FWHT, PSNR, MSE, SSIM, NC

\section{INTRODUCTION}

In this digital era, exchange of information through internet has been increasing at very sharp pace because it is an inexpensive and rapid way to communicate. A lot of information is being conveyed digitally through this faster channel than any other medium. Despite of various blessings of this digital medium such as mobile accessibility from anywhere, online storage, distribution and modification of data, still there are few flaws in this system which can question the authenticity, integrity, availability and confidentiality of data. So data needs to be secured from swindling and deceitful actions trying to manipulate and steal the data without authorization [1][3]. Digital image watermarking is used to hide the data into image, this data is called watermark it could be image or text embedded into original image for authenticity of this original image. Operation of embedding watermark in original image and retrieving watermark from watermarked image are shown in figure1.1 and 1.2 respectively. Original image and watermark image are manipulated in watermarking to get watermarked image with minimum alteration in original image's visual quality. A watermark could be retrieved from watermark image by using defined extraction method. After watermarking embedding, of original image quality is measured using some parameters like PSNR, MSE. Values of these parameters are calculated by using difference between original image and Image formed after performing watermarking [7]. Watermarking algorithm may be blind or non blind. If the operation for fetching watermark from original image needs watermark then it will be called a non blind procedure of watermarking. If watermark can be retrieved without original data then it will be called blind procedure of watermarking [3].

\section{LITERATURE SURVEY}

Since last decade digital image watermarking is one of the most researched area, various researcher have proposed techniques in almost all domains. Ray et al.(2015) In presented paper, they developed a technique for watermarking in which they encrypted the values of watermark image obtained from SVD using RSA encryption algorithm and then they embedded these encrypted values into original image in order to increase the security of algorithm[6]. Gunjal et al.(2015) They developed a non blind technique for watermarking based on multi objective evolutionary optimizer (MEO) using DWT and SVD. Haar wavelet is used for DWT decomposition of image into sub-bands. Multi objective evolutionary optimization through genetic algorithms has been a core of this paper. Genetic algorithms help in choosing of wavelet with better performance in less time. They have used Fibonacci lucas transform to make watermark image scrambled for security purpose[7]. Chauhan et al.(2014) In this paper, they proposed a image watermarking detection technique using RGB intensity and PSNR Value. RGB intensity and PSNR Value watermarking technique is realized by using two watermark images[15].

\section{WATERMARKING TECHNIQUES}

There are several techniques for embedding watermark in spatial domain as well as in transform domain. In this paper, we have proposed a scheme based on transform domain.

\subsection{Discrete Wavelet transform (DWT)}

It is used to divide image in different parts using wavelets of different frequencies [8].Discrete wavelet transform will decompose image into bands LL, HL, LH and HH. At next level a single band could be decomposed further up to any level. Haar Wavelet is widely preferred in watermarking. To get back the original image after decomposition inverse discrete wavelet could be applied [8].

Haar wavelet is discontinuous, and resembles a step function. Haar wavelet is called as the mother of all wavelets as it produces all wavelet functions used in transformation through translation and scaling functions [9]. 


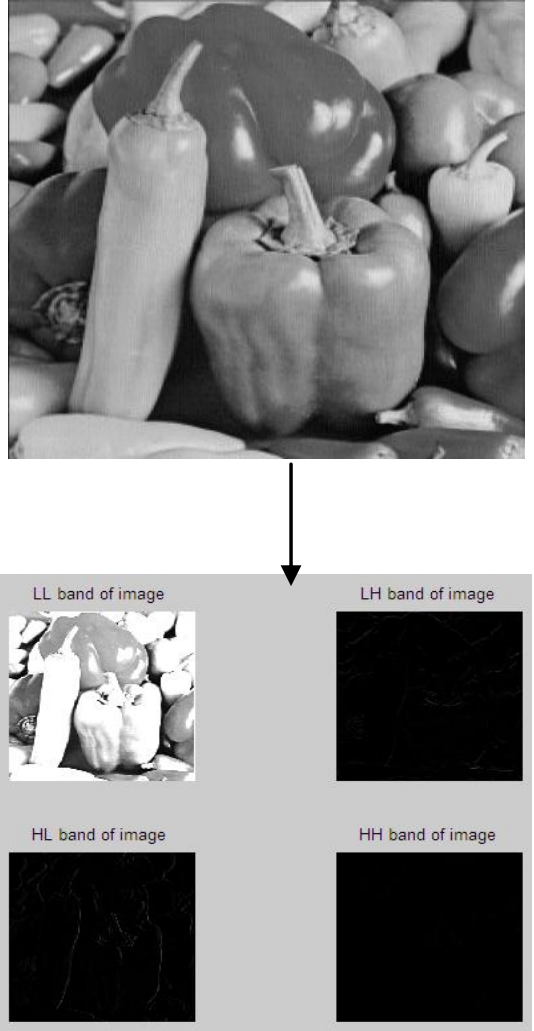

Fig 1 Dissolution of an Image

\subsection{Singular Value Decomposition (SVD)}

SVD is a mathematical tool which decomposes a matrix. Matrix is divided into three matrices, two orthogonal matrices and one diagonal matrix. A be an image matrix. After SVD, A could be written as [9][13] :

$$
\begin{gathered}
\mathrm{A}=\mathrm{U}_{\mathrm{A}} \mathrm{S}_{\mathrm{A}} \mathrm{V}_{\mathrm{A}}^{\mathrm{T}} \\
\mathrm{U}_{\mathrm{A}}=\left[\mathrm{u}_{1}, \mathrm{u}_{2}, \ldots \ldots \ldots \ldots, \mathrm{u}_{\mathrm{N}}\right] \\
\mathbf{V}_{\mathrm{A}}=\left[\mathrm{v}_{1}, \mathrm{v}_{2}, \ldots \ldots \ldots, \ldots, \mathrm{v}_{\mathrm{N}}\right] \\
\mathrm{S}_{\mathrm{A}}=\left(\begin{array}{ccc}
\mathrm{S}_{1} & \ldots . . & \ldots . . \\
\ldots & \mathrm{s}_{2} & \ldots \\
\ldots & \ldots . . & \mathrm{s}_{3}
\end{array}\right)
\end{gathered}
$$

$\mathrm{S}_{\mathrm{A}}$ is a diagonal matrix of singular values. These diagonal values are replaced with diagonal values of watermark. SVD is used in watermarking because singular values are more stable and does not change much after embedding process [10].

\subsection{Fast Walsh Hadamard Transformation (FWHT)}

The Walsh-Hadamard transform decomposes a signal into a number of functions that are rectangular or square waves with values of +1 or -1 . These basis functions are non-sinusoidal, orthogonal transformation technique. For 2-D images FWHT coefficients are calculated by first row wise then column wise [10]. The 4X4 Hadamard matrix is defined as $\mathrm{H} 1$ is given as [18]

$$
\mathbf{H}_{2}=\frac{1}{2}\left[\begin{array}{rrrr}
1 & 1 & 1 & 1 \\
1 & -1 & 1 & -1 \\
1 & 1 & -1 & -1 \\
1 & -1 & -1 & 1
\end{array}\right]
$$

\subsection{Encryption of Watermark Image}

Watermark image has been encrypted before embedding using key based encryption algorithm. This algorithm generates key matrix according to the size of watermark image and encrypt every pixel with different key value. This key is saved to be used in decryption process.

\section{PROPOSED ALGORITHM}

In existing schemes DWT and SVD are used in several ways along with other techniques such as DCT and FWHT. In this scheme we used a DWT and SVD along with FWHT but in a manner to embed watermark in all of sub bands to achieve high capacity and imperceptibility. Watermark is encrypted before embedding and decryption is performed on extracted watermark.

\subsection{Watermark Embedding Scheme}

Step 1: Load a color cover image of size 1024×1024 .

Step 2: Find its RGB components and choose green component for embedding, otherwise do nothing.

Step 3: Apply DWT 1-level haar wavelet on image to get subbands such as LL, LH, HL, HH as shown in figure 4.1

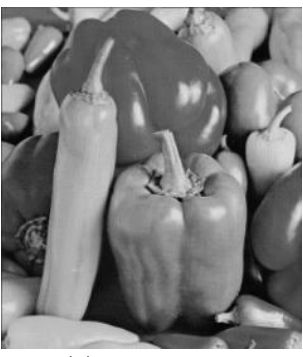

(a)

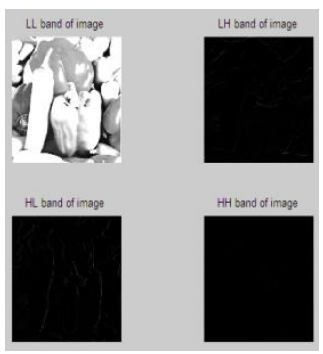

(b)
Fig 2 DWT decomposition. (a) Pepper image,

(b) 1level Haar DWT

Step 4: Apply DWT 1-level haar wavelet on each of these sub-bands of image for further decomposition as shown in figure 4.2. Select $\mathrm{HH} 1, \mathrm{HH} 2, \mathrm{HH} 3$ and $\mathrm{HH} 4$ sub-bands for embedding watermark and apply FWHT on each of these.

\begin{tabular}{|c|c||c|c|}
\hline LL1 & HL1 & LL3 & HL3 \\
\hline LH1 & HH1 & LH3 & HH3 \\
\hline \hline LL2 & HL2 & LL4 & HL4 \\
\hline LH2 & HH2 & LH4 & HH4 \\
\hline
\end{tabular}

Fig 3 DWT decomposition used in proposed technique 
Step 5: Apply SVD on these sub-bands to get singular values.

Step 6: Load gray scale watermark image of size 256x256 and apply SVD on it to get its singular values after encrypting it.

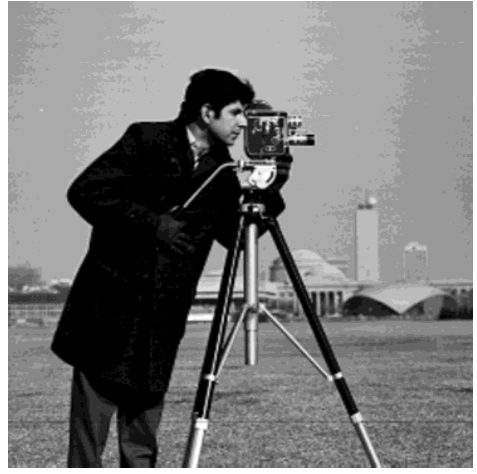

(a)

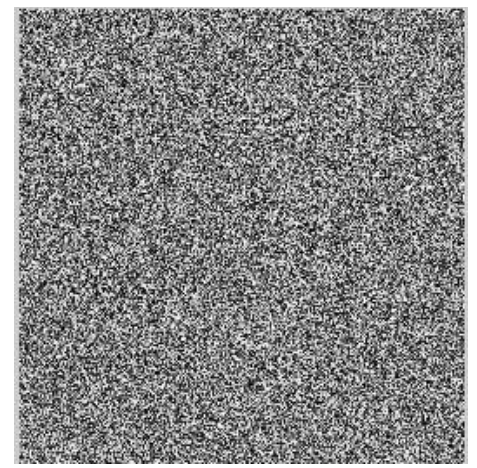

(b)

Fig 4 (a) original watermark, (b) encrypted watermark

Step 7: Modify singular values of each sub-band with singular values of watermark image using equation given below:

$$
S_{\mathrm{ii}}=\mathrm{S}+\left(\mathrm{S}_{\mathrm{i}} * \alpha\right)
$$

Where $\alpha$ is a scaling factor having value between 0 and 1 .We have taken $\alpha=0.003, \mathrm{~S}_{\mathrm{ii}}$ are modified singular values, $\mathrm{S}$ represents the singular values of cover image and $S_{i}$ are singular values of watermark image.

Step 8: Apply inverse SVD on each of four sub-bands using modified singular values.

Step 9: Apply inverse FWHT on each sub-band and inverse DWT haar wavelet to get the full image.

Step 10: Show the watermarked image and calculate the values of PSNR, MSE and SSIM.

\subsection{Watermark Extracting Scheme}

Step 1: Load a watermarked image and check it if it is color image or gray scale image.

Step 2: If image is color then find its RGB components and choose green component for extracting watermark, otherwise do nothing.

Step 3: Apply DWT 1-level haar wavelet on image to get subbands such as LL, LH, HL, HH and apply DWT 1-level haar wavelet on each of these sub-bands of image for further decomposition as shown in figure 4.2

Step 4: Select HH1, HH2, HH3 and HH4 sub-bands and apply FWHT on each of these.

Step 5: Apply SVD on these sub-bands to get singular values.

Step 6: Extract singular values from each sub-band with singular values of watermark image using equation given below:

$$
S_{\mathrm{iii}}=\frac{\left(S_{\mathrm{ii}}-\mathbf{S}\right)}{\alpha}
$$

Where $\alpha$ is a scaling factor having value between 0 and 1 .We have taken $\alpha=0.003, S_{i i}$ are modified singular values, $S$ represents the singular values of cover image and $S_{\text {iii }}$ are singular values of extracted watermark image.

Step 7: Apply inverse SVD on four of these extracted singular values to get the encrypted watermark image, and show four extracted watermark after decryption. 1

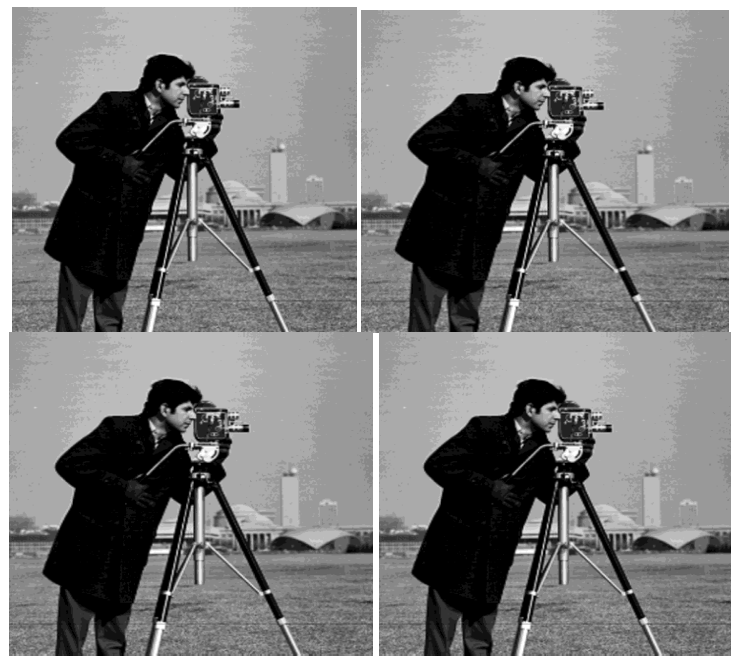

Fig 5 Extracted watermarks from all four sub-bands

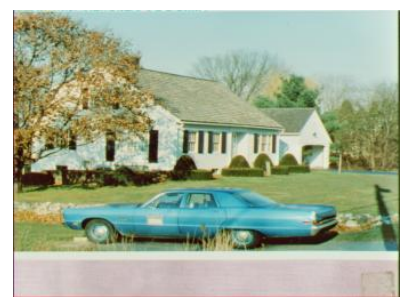

(a)

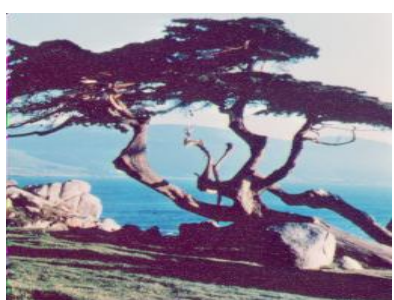

(b)

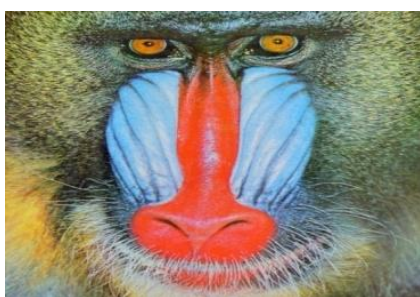

(c) 


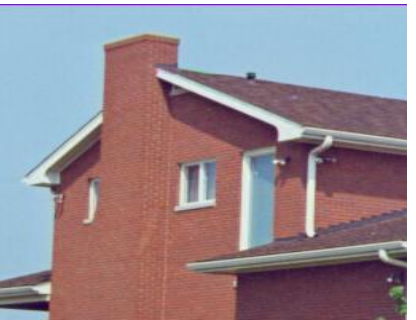

(d)

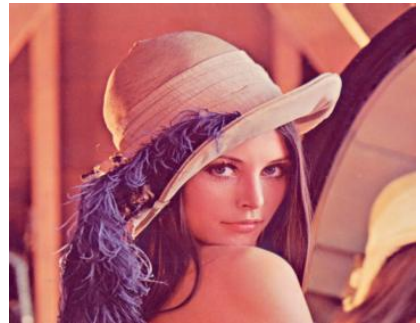

(e)

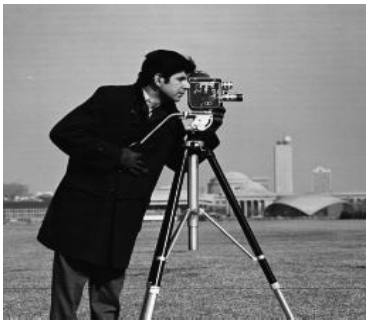

(f)

Fig 6 Different testing images (a) car image, (b) lake image, (c) baboon image, (d) house image, (e) lena image, (f) cameraman (watermark image)

\section{RESULTS}

\subsection{Performing Measures}

\subsubsection{Peak Signal to Noise Ratio}

The peak signal to noise ratio is used to measure imperceptibility of watermarked image with respect to original cover image. Higher value of PSNR implies the good perceptual quality of image and is measured in $\mathrm{dB}$ (decibel) [2]. It is given as:

$$
P S N R=10 \cdot \log _{10}\left(\frac{M A X_{i}^{2}}{M S E}\right)
$$

Where MSE is Mean square error, which is given as:

$$
M S E=\frac{1}{m n} \sum_{0}^{m-1} \sum_{0}^{n-1}\|f(i, j)-g(i, j)\|^{2}
$$

Where $f(i, j)$ and $g(i, j)$ are pixel values of original image and watermarked image, respectively [9].

\subsubsection{Normalized correlation}

Normalized correlation is a parameter to check the robustness of watermarking scheme. $\mathrm{NC}$ is used to measure similarity between extracted watermark and original watermark[9]. It is given as:

$$
N C=\frac{\sum_{k=1}^{n} w_{1} w_{2}}{\sqrt[2]{\sum_{k=1}^{n} w_{1}} \sqrt[2]{\sum_{k=1}^{n} w_{2}}}
$$

Where $\mathrm{w}_{1}$ is embedded watermark and $\mathrm{w}_{2}$ is extracted watermark.

\subsubsection{Structural Similarity Index (SSIM)}

SSIM index is a method for measuring the similarity between two images. The resultant SSIM index is a decimal value between -1 and 1 , and value 1 is only reachable in the case of two identical sets of data. More similar images more similar will be the SSIM value. It is given as:

$$
\operatorname{SSIM}(x, y)=\frac{\left(2 \mu_{x} \mu_{y}+c_{1}\right)\left(2 \sigma_{x y}+c_{2}\right)}{\left(\mu_{x}^{2}+\mu_{y}^{2}+c_{1}\right)\left(\sigma_{x}^{2}+\sigma_{y}^{2}+c_{2}\right)}
$$

\subsection{Imperceptibility}

Imperceptibility of watermarked image is calculated by calculating parameters such as PSNR, MSE and SSIM by comparing watermarked image with original cover image. When we need to achieve high embedding capacity then imperceptibility of watermarked image falls because they are contrary to each other. In our purposed scheme, we have achieved a high capacity and still have good imperceptibility shown in tables after performing techniques on various test images.

\section{Table 1 PSNR, SSIM and MSE values for color images}

\begin{tabular}{|l|l|l|l|}
\hline Images & MSE & SSIM & PSNR \\
\hline Car & 4.4160 & 0.9903 & 38.1475 \\
\hline Lena & 3.8687 & 0.9970 & 38.4841 \\
\hline Baboon & 11.7126 & 0.9812 & 32.9633 \\
\hline Lake & 7.3452 & 0.9864 & 36.2351 \\
\hline House & 2.1524 & 0.9935 & 41.3448 \\
\hline
\end{tabular}

\subsection{Robustness}

To check the robustness of proposed scheme watermarked image is subjected to many attacks. Extracted watermarks from infected image are compared with original one to calculate $\mathrm{NC}$ values as shown in table 4 . Value of $\mathrm{NC}$ varies between 0 and 1, if we calculate $\mathrm{NC}$ for same images, value of $\mathrm{NC}$ will be equal to 1 . NC values shown in table 2 imply that our proposed scheme makes all of four embedded watermarks almost equally robust against several attacks. Graph compares the effect of various attacks on watermarked image on the basis normalized correlation for color images. Graphs show that all the sub-bands we used in embedding process are equally robust against all these noise adding attacks. These attacks have been applied at different density shown in tables.

Table 2 NC values after different attacks on color images

\begin{tabular}{|l|l|l|l|l|l|}
\hline \multirow{2}{*}{ Attacks } & \multirow{2}{*}{ Density } & \multicolumn{4}{|l}{$\begin{array}{l}\text { NC values for Extracted watermarks } \\
\text { from all of four bands }\end{array}$} \\
\cline { 3 - 6 } & & $\mathrm{HH} 1$ & $\mathrm{HH} 2$ & $\mathrm{HH} 3$ & $\mathrm{HH}$ \\
\hline No Attack & & 0.9895 & 0.9875 & 0.9892 & 0.9905 \\
\hline $\begin{array}{l}\text { Gaussian } \\
\text { noise attack }\end{array}$ & 0.001 & 0.7426 & 0.7418 & 0.7426 & 0.7417 \\
\cline { 2 - 6 } & 0.005 & 0.7426 & 0.7427 & 0.7423 & 0.7417 \\
\hline $\begin{array}{l}\text { Salt } \\
\text { pepper } \\
\text { attack }\end{array}$ & 0.005 & 0.7865 & 0.7852 & 0.7843 & 0.7846 \\
\cline { 2 - 6 } & 0.001 & 0.8227 & 0.8164 & 0.8164 & 0.8121 \\
\hline $\begin{array}{l}\text { Speckle } \\
\text { attack }\end{array}$ & 0.001 & 0.8114 & 0.8146 & 0.8124 & 0.8107 \\
\cline { 2 - 6 } & 0.005 & 0.7854 & 0.7777 & 0.7785 & 0.7741 \\
\hline Poison & & 0.7793 & 0.7765 & 0.7766 & 0.7759 \\
\hline
\end{tabular}




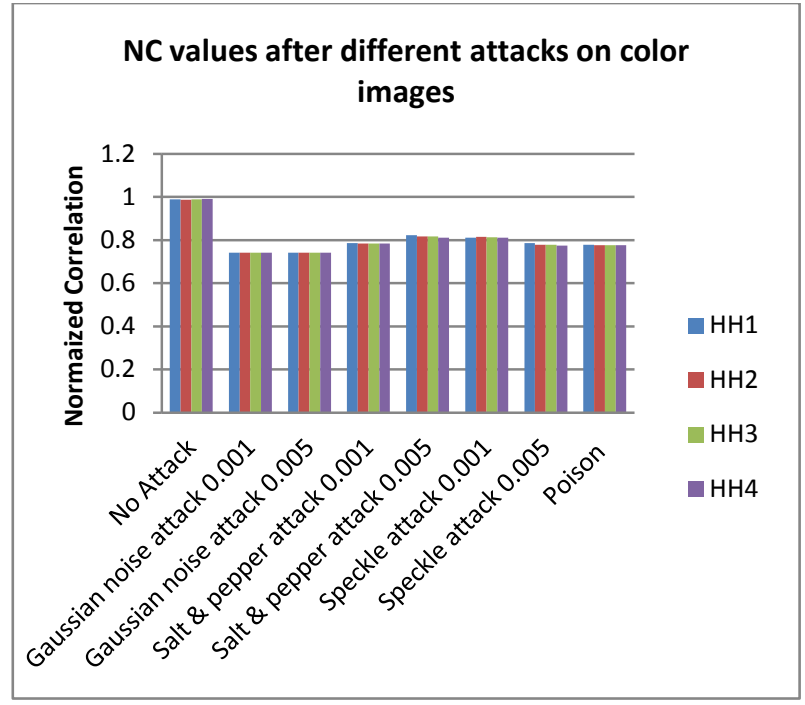

Graph 1 Comparison of normalized correlation values under different attacks on color image

\section{CONCLUSION AND FUTURE WORK}

Proposed watermarking scheme for embedding watermark in all of four sub-bands (LL, LH, HL, HH) has achieved watermarked image with high perceptual quality. It has achieved PSNR value above $30 \mathrm{db}$ which implies that cover image has not lost its originality. SSIM has also been calculated to show similar structure of original image and watermarked image. Proposed scheme has also been subjected to various noise adding attacks and results show robustness of scheme against these attacks.

For future work this technique can be used with other techniques like CWT (complex wavelet transform) and SWT (stationary wavelet transform). Further, this technique can be extended for audio/video watermarking.

\section{REFERENCES}

[1] I. J. Cox-t, J. Kiliant, T. Leightont, and T. Shamoont, "Secure spread spectrum watermarking for images, audio and video," in Proc. IEEE, pp. 243-246, 1996

[2] H. Kaur, "Study on audio and video watermarking," International Journal of Communication Network Security ISSN: $2231-1882$, Volume-2, Issue-1, pp. 3438,2013

[3] G. Chawla, R. Saini, R. Yadav, Kamaldeep, "Classification of Watermarking Based upon Various Parameters," International Journal of Computer Applications \& Information Technology Vol. I, Issue II, pp. 17-19, 2012

[4] M. Abdullatif, A. M. Zeki, J. Chebil, T.S.Gunawan, "Properties of Digital Image Watermarking," IEEE 9th International Colloquium on Signal Processing and its Applications, pp. 235-240, 2013

[5] M. Keyvanpour, F. M. Bayat, "A New Encryption Method For Secure Embedding In Image Watermarking," 3rd International Conference on Advanced Computer Theory and Engineering(1CACTE) pp. $403-407,2010$
[6] A. K. Ray, S. Padhihary, P. K. Patra and M. N. Mohanty, "Development of a new algorithm Based on SVD for image watermarking," Computational Vision and Robotics, Springer India, pp. 79-87, 2015

[7] B. L. Gunjal and S. N. Mali, " MEO based secured, robust, high capacity and perceptual quality image watermarking in DWT-SVD domain, " SpringerPlus, pp. $1-16,2015$

[8] Y. R. Rao, A. Vikram, “ Reliable RGB Color Image Watermarking using DWT and SVD ," International Journal of Scientific Engineering and Technology Research Volume.04, IssueNo.20, pp. 3797-3801,2015

[9] N. Goel , G. Singh , "Study of Wavelet Functions of Discrete Wavelet Transformation in Image Watermarking , " An International Journal of Engineering Sciences, pp.154-160, 2015

[10] G. cetinel , L. cerkezi, "Chaotic Digital Image Watermarking Scheme Based on DWT and SVD, 9th International Conference on Electrical and Electronics Engineering (ELECO) , pp. 251-252,2015

[11] R. Islam and J. M. Kim, "Reliable RGB color image watermarking using DWT and SVD," in Proc. IEEE Int Conf. Informatics Electronics Vision, Dhaka, Bangladesh, pp. 1-4, 2014

[12] S. Lagzian, M. Soryani, M. Fathy, "Robust watermarking scheme based on RDWT-SVD:Embedding Data in All subbands," Artificial Intelligence and Signal Processing (AISP), 2011 International Symposium on, pp. 48-52, 2011

[13] V. S. Jabade and S. R. Gengaje, "Literature review of wavelet based digital image watermarking techniques," Int. Journal of Computer Applications, Vol. 31, no.1, pp. 28-35, 2011.

[14] C. Song, S. Sudirman, M. Merabti, "Analysis of digital image watermark attacks," in Proc. IEEE Int. Conf. Consumer Communications and Networking, Las Vegas, United States, pp. 1-5, 2010.

[15] N. Goel , G. Singh , “ Entropy Based Image Watermarking using Discrete Wavelet Transform and Singular Value Decomposition , "3rd 2016 International Conference on "Computing for Sustainable Global Development", 16th - 18th March, 2016

[16] N. Chauhan, A. A. Waoo, P.S.Patheja, Information hiding watermarking detection technique by PSNR and RGB intensity , "Journal of Global Research in Computer Science, 3 (9), pp. 18-22,2012

[17] C.C. Lai, “ An improved SVD-based watermarking scheme using human visual characteristics". Opt. Commun. 284(4), pp. 938-944,2015

[18] K. Meenakshi, C. S. Rao, K.S. Prasad, "A Robust watermarking scheme based on Walsh-Hadamard Transform and SVD using Zig-Zag Scanning" 13th International Conference on Information Technology, pp. 167-172, 2014 